\title{
Research on Crisis Perception and Prevention of Enterprise Human Resources
}

\author{
Lili Lu \\ Xi’an International University, Xi'an, Shaanxi, 710077
}

Keywords: Crisis Perception, Enterprise Human Resource, Prevention Method

\begin{abstract}
The rise and fall of enterprises reflects the rise and fall of the level of enterprise management, and corporate human resources are restricting the level of management. With the increasing competition in China's market and the continuous expansion of the scale of enterprises, human resources crisis has been more and more people's attention. Based on the theory of human resource management, this paper focuses on the management of human resource crisis management and the management of human resources crisis and the research on the perception and prevention of human resource crisis. In view of the shortcomings of the research in this field, this paper gives the definition of human resource crisis, the use of the management of science technology and methods, through the human resources crisis from the table and analysis and comprehensive human resource crisis trigger mechanism and human Resource crisis perception model, and through the empirical study to verify the perception model. Then the method of human resource crisis evaluation is put forward according to the conclusion of perceptual research, and the prevention system based on crisis source blocking is constructed.
\end{abstract}

\section{Introduction}

As we all know, human resources is one of the most important resources of enterprises, almost all the work of the enterprise is done by the people, the quality of work, the level of efficiency depends on the quality of the quality of employees, enterprises can adapt to environmental changes, Whether the market competition in a favorable position, the key is whether the enterprise has high-quality staff. To become a first-class enterprise, the key is to have first-class talent. Therefore, the business community often has such a saying, "would rather have a first-class talent, but only second-rate technology companies, better than investing first-class technology, but only second-rate talent company." The most important job of the enterprise is to do a good job in the management of human resources, to establish a mechanism and atmosphere conducive to attracting talents, retaining talents and motivating talents to prevent the loss of talents, thus avoiding the crisis caused by the mismanagement of human resources. In today's complex market environment, with the intensification of market competition and the arrival of knowledge economy enterprises are facing by the external environment and internal factors caused by the heavy crisis, and more and more types of crisis, the performance of the enterprise had a serious impact. Crisis of the complex, enough to allow enterprises to lose the accuracy of the crisis is serious enough to allow enterprises to face the situation of life and death. In the enterprise is facing or potential of the various crises, the human resource crisis is more subtle, but also enterprises are often easy to overlook. By the zero survey company, Tsinghua University School of Public Administration Crisis Management Group and China Hewlett-Packard Co., Ltd. to work together to complete the "corporate crisis management status" research shows that China's state-owned enterprises have near the existence of human resources crisis, and some state-owned enterprises That the human resources crisis has had a serious impact on its business, the state-owned enterprises have experienced or are experiencing a high turnover rate of staff caused by human resources crisis, and experienced or are experiencing the high-level management personnel accidental leave caused by the crisis The proportion of state-owned enterprises is as high as that of foreign enterprises and private enterprises. It can be seen that the loss of senior management personnel is an important manifestation of the human 
resource crisis. In addition, the human resource crisis also includes the sudden and resignation of the main backbone of the enterprise, the apparent loopholes in the payroll system, the loss of key employees, and even the more extreme and serious phenomena in the personnel crisis. Chinese enterprises are neither the lack of funds, nor technology, but not the market, but talent, especially the outstanding senior management personnel.

\section{The Mechanism of Human Resource Crisis}

From the impact of the environment on the human resource crisis. Often, changes in employee status are influenced by factors such as personal factors, macroeconomic environment, and external environment. Enterprises in the process of achieving its strategic development goals, when the enterprise's development strategy, corporate culture, organizational structure and management of any one of the changes in the way, will affect the staff of their organization's attitude, this time, the staff will According to their own value positioning and value expectations to adjust his attitude to the enterprise to adapt to the environment. When the staff can not adapt to the organizational environment, he will appear rebellious. At this point, employees want managers to find his attitude changes, and by adjusting the external environment to stabilize its attitude to the enterprise, if the organizational environment can not be adjusted in time, it will lead to staff dissatisfaction with the work, the work of negative slack, Or even choose to leave. At this point, the overall situation of the staff began to deteriorate. If summed up from this point of view, the human resource crisis behavior is due to the organizational environment is not satisfied, not suited to the trigger.

From the managers of the crisis caused by the role of view. Speaking of the organizational environment in front of a great impact on the attitude of the staff, but the environment is only a medium, the real attitude of the staff to play a decisive impact is the organization's management model and methods. The characteristics of the subjectivity of the human resource crisis tell us that managers and policymakers are actors who have caused changes in organizational environment. They have played a key role in improving the organizational environment and alleviating employee dissatisfaction. Therefore, the attitude of employees to the organizational environment of its essence can also be equivalent to its organizational management model and method of attitude. After all, management behavior and organizational environment is the same time affect the individual employees, and individual employees on the organizational environment is the views of the views of managers. From this point of view, the human resource crisis behavior is due to the management system is imperfect, management mode and method of unreasonable, as well as the organizational environment of the results of the role of disharmony.

Human resource crisis is not a sudden occurrence, it needs to experience from the implicit form to the explicit shape of the transformation process. When the human resource crisis manifests itself as implicit form, the human resource crisis process begins. This process is usually going through four stages of complaint, burnout, resistance and separation. When employees are not satisfied with the matter, will be some way to this negative emotions to vent out, its purpose is to attract the attention of managers, provided that managers can solve the problem. In the complain stage, although the staff feel dissatisfied, but did not leave the intention, the task can be completed normally. If the manager does the appropriate intervention, everything will return to normal However, if the manager of the laissez-faire, or if taken measures but did not meet the psychological expectations of employees, will lead to emotional disappointment and frustration, and then Produce job burnout. In the job burnout state of the staff, negative attitude, lazy behavior, but also often find someone else's problems. Their performance at this time has a greater degree of deviation from the normal track, its performance has had a greater impact.

\section{Human Resources Crisis Prevention Strategy}

For the staff of cultural incentives, through advocacy, encourage a conducive to creativity and development of the values to create a good interpersonal environment, so that each employee can feel comfortable, enthusiastically put into work. Therefore, we should focus on creating an 
organizational culture system with "core management" as the core, forming advocacy ability, supporting creativity and respecting each other. We can increase the flexibility of internal structure and system and increase the scope of personal activities. Creative activities use different perspectives and different sources of information, and keep the organization open. Employees in such a cultural environment, the creation of potential will be excited and active, access to extraordinary release and play, in order to stimulate the vitality of the entire organization.

Clear and reasonable organizational structure is the prerequisite for enterprises to create a flexible working environment, but also its essential a soft management infrastructure. In the era of knowledge economy, enterprises should change the organizational structure of the traditional pyramid organization with a less hierarchical network type. The traditional management of the organizational structure is pyramid type, the organization level too much, the information channel is too long, slow response to the functional departments of mutual isolation, information flow between the upper and lower levels of information often distorted, distortion. Flexible management of the enterprise is the level of small, to the network-based flat organizational structure instead of multi-level vertical organizational structure, improve the efficiency of information transmission and work efficiency, strengthen the communication between departments, improve the overall response sensitivity of the enterprise, So that enterprises can quickly seize market opportunities.

Business objectives focus on the start of the capital to solve the problem and the market, organizational structure is not clear, lack of funds, training, less time, time constraints, training needs is not urgent, can not develop a long-term training program, the enterprise can not start a general training activities, from Cost and efficiency considerations, the traditional mentoring way to carry out business technology and market promotion and other aspects of small-scale focus on staff training is ideal.

The In this period, the enterprise funds are not sufficient, the main business process and organizational structure is not stable, job responsibilities are not clear, there are often one person or the role of cross-cutting phenomenon, leading employees are often entrepreneurial enthusiasm, rather than reputation and formal status The Therefore, the focus of corporate compensation should be placed on the external competitiveness of compensation, dilute the internal equity of pay. The overall salary rigidity should be smaller, that is, the proportion of basic wages and benefits is small, and the proportion of performance bonuses is large. At the same time relative to the wages, welfare and other material incentives, the achievements of incentives, job incentives, motivations and other incentives to stimulate the use of more frequent, the usual practice is to make commitments to employees or agreement, with equity, future earnings or future positions Such as long-term incentives to replace the current high salary.

Due to the lack of a clear organization and formal rules and regulations of the management and constraints of the management of the staff is more casual and loose, mostly by the entrepreneur to manage the individual employees, entrepreneurial personal emotions and hobbies will directly affect the Staff management, so to strengthen the entrepreneur's personal legal awareness, emphasizing mutual respect, equality and mutual assistance. During this period, the scale of the enterprise changed greatly, the organizational structure began to be clear, the enterprise entered the standardized management stage, the human resources management department can set up by the departmental management personnel and human resources experts to recruit the recruitment team. Due to the expansion of the product or service market, the human resources department should focus on the recruitment of production personnel and sales staff, emphasizing the personal experience of the candidates and the customer source. Enterprises have the funds to support advertising costs, you can choose to influence the media and key universities, managers can search through the headhunting company, save the company a lot of recruitment and selection time. In the recruitment process, pay attention to establish the image of enterprises, expand the influence and visibility of enterprises.

Corporate profits increase, on the one hand began to increase the basic wage and increase the benefits of the other hand, because the company is in a positive state of expansion, to encourage individual contributions, according to individual performance and performance bonuses accounted 
for a large proportion. During this period many enterprises to further increase investment, therefore, the company's cash stock is often not much. In order to attract high-level talent to join, companies should also emphasize the importance of long-term incentives. In addition, due to the emergence of new and old employees of the differentiation, the pay will have a greater difference, this stage of the incentive program focused on strengthening the sense of fairness within the enterprise, enhance the competitiveness of the compensation market, design diversified incentives to meet different levels the needs of the staff.

\section{Conclusion}

From the conclusion of human resource crisis factor analysis, it can be found that the human resource crisis is mainly due to the deviation from the expectation of employee expectation and the actual return, so it is impossible to reflect the degree of deviation from the subjective judgment index and evaluate the human resource crisis status. Therefore, the crisis evaluation part of the enterprise human resources-related financial indicators as a basis for human resource crisis prediction, the empirical analysis of the key management links may lead to the crisis signs summarized, the collection of human resources crisis evaluation indicators using AHP and fuzzy mathematics The risk rating model is combined with the degree of human resource crisis to design the crisis barometer, divide the crisis level, and provide the basis for the prevention and control measures.

\section{References}

[1] Ding Juanjuan, Zheng Chunmei. Establishment of Early Warning System of Talent Loss in High and New Technology Enterprises [J]. Commercial Research. 2008 (02)

[2] Chen Xinde, Zhou Jin. Human Resource Management Risk Early Warning Model Based on Fuzzy Comprehensive Evaluation Method [J]. Commercial Research. 2006 (15)

[3] Yang Yuekun. To build the enterprise "Noah's Ark" - Enterprise crisis identification and prevention countermeasures [J]. Special zone economy. 2006 (07)

[4] Zhang Wen, Linda Duxbury, Li Li. Chinese employees "work / life balance" of the theoretical framework [J]. Modern Management Science. 2006 (05)

[5] Yao Xinzhuang. Human resource management crisis coping strategy [J]. Human Resources Development. 2006 (04)

[6] Xu Bo, Pang Li. Crisis Management for Brain Drain [J]. Journal of Jingmen Vocational \& Technical College. 2006 (01) 